\title{
Dynamical models of movement coordination
}

\author{
P.J. Beek ${ }^{\text {a,b,c, } * \text {, C.E. Peper }}{ }^{\text {a,b }}$, D.F. Stegeman ${ }^{\text {a,d }}$ \\ ${ }^{\mathrm{a}}$ Institute of Fundamental and Clinical Human Movement Sciences, Amsterdam / Nijmegen, The \\ Netherlands \\ ${ }^{\mathrm{b}}$ Faculty of Human Movement Sciences, Free University, Van der Boechorststraat 9, 1081 BT Amsterdam, \\ The Netherlands \\ ${ }^{c}$ Center for the Ecological Study of Perception and Action, University of Connecticut, Storrs, USA \\ ${ }^{d}$ Institute of Neurology, University of Nijmegen, P.O. Box 9101, 6500 HB Nijmegen, The Netherlands
}

\begin{abstract}
This article examines the status of dynamical models of movement coordination qua phenomenological models. After a brief outline of the aims, methods and strategic assumptions of the dynamical systems approach, a survey is provided of the theoretical and empirical progress it has made in identifying general principles of coordination. Although dynamical models are constructed for phenomena at a particular level of analysis for which they provide descriptive explanations, their dynamics can sometimes be linked to or associated with the dynamics of processes at other levels of analysis. The article concludes with a tentative scheme to clarify the position of the dynamical approach relative to other extant approaches in movement science.
\end{abstract}

Bodily movements occur in the context of the everyday functioning of people while realizing specific task goals. As a rule, such movements involve the participation of multiple joints and limbs. When in action, these body parts are coordinated, that is, they are brought into proper relation to one another as well as to the surrounding layout of surfaces (cf. Turvey, 1990). To the naked eye, this coordination may look relatively simple, as in picking up an object, or relatively complicated, as in juggling, performing an attacking forehand drive in table tennis or playing the drums. To the

\footnotetext{
* Corresponding author. Tel: + 3120444 8532. Fax: +31 20444 5867. E-mail: P_J Beek@FBW.VU.NL.
} 
movement scientist, however, all coordination is complex in that he or she is confronted with the challenge to explain coordinated movements as the orderly products of a hybrid biological organization involving a very large number of different subsystems (e.g., vascular, neural, muscular, skeletal). These subsystems are operating at different rates and are connected in intricate ways. Due to this compositional complexity, the problem of movement coordination is extremely difficult to resolve in a scientifically satisfactory way. Finding an adequate solution is hampered by the fact that the field of motor control is still very much partitioned according to the traditional disciplines of movement science (mechanics, neurophysiology, psychology, and so on), whereas a multidisciplinary or interdisciplinary approach is required.

Broadly speaking, two types of approaches may be distinguished in movement science: structural and phenomenological approaches (cf. Otten, 1991). Structural approaches seek causal explanations of movement in terms of dedicated structures within the human body. Phenomenological approaches, in contrast, seek noncausal explanations in terms of phenomenological laws and principles without reference to dedicated mechanisms and structures within the human body.

Structural models of motor control are typically (neuro)physiological models which attempt to explain different aspects of motor behaviour on the basis of hypothetical (neuro)physiological mechanisms for the generation of movement (such as an efference copy, an oculomotor coupling, a mechanism for the control of threshold values of the stretch reflex, and so forth). The aim of these models is to elucidate the structural basis of functional properties, while phenomenological models aim at describing functional properties without addressing the structural basis of these properties. Thus, all models that do not make explicit propositions with regard to the neural mechanisms of motor control, but rather attempt to find an appropriate mathematical description of the kinematics of movement belong to this class of models. Usually, the aim of such mathematical descriptions is to derive hypotheses with regard to the variables that might play a central role in the neural control of movement and the principles on which this control might be based. Our concern in this article is with a special subclass of phenomenological models of movement coordination, namely (nonlinear) dynamical models. Dynamical systems theory is currently penetrating the sciences at large, including the traditional disciplines of movement science, and holds the promise, as we shall see, of providing a general, interdisciplinary theory of the formation of spatiotemporal pat- 
terns at the level of movement coordination itself as well as at the level of the corresponding neural events.

In the present contribution we examine the current status of dynamical models of movement coordination qua phenomenological models. We use the term "status" here in a broadly and loosely defined way. We are interested in questions such as: What is the nature of dynamical models? How are thcy derived and what do they contribute to our understanding? How do dynamical models for movement develop, and in what sense are they empirically progressive? What is the explanatory value of these models, and what are their limitations? How are we to think of dynamical models in relation to other kinds of models?

We hope that an attempt to provide accurate answers to these questions will help those who are working from other perspectives than dynamical systems theory in appreciating the contributions of this approach and in comparing these contributions with contributions made by other approaches. In this regard, this article has the character of a review of the dynamical systems approach, although it is not simply a tutorial ${ }^{1}$. It raises several controversial points that are aimed at stimulating discussion among movement scientists in general about the sort of models that are required to come to terms with the problem of movement coordination.

Given these aims, the article is structured as follows. First, a brief outline is provided of the general goal of the dynamical systems approach and the strategic assumptions and methods it has adopted in the pursuit of this goal. Subsequently, a survey is provided of the main empirical and theoretical findings of the approach in the field of movement. This survey is not meant as an exhaustive list of achievements but rather as an illustration of the manner in which progress is being made in this particular programme of research, how research builds on previous findings, what kind of insights are obtained, and so on. This survey is followed by a section devoted to a discussion of the explanatory value of dynamical models of coordination and two sections on the relationships between dynamics and neurophysiology and between dynamics and biomechanics, respectively. The article concludes with a tentative conceptual scheme that clarifies the position of the dynamical approach relative to other approaches.

\footnotetext{
${ }^{1}$ Excellent tutorials on the dynamical systems approach to movement coordination are provided by Kelso and Schöner (1988), Schöner and Kelso (1988c), and Kelso, Ding and Schöner (1992).
} 


\section{Brief characterization of the dynamical systems approach}

Nonlinear dynamics is a branch of mathematics that deals with the formal treatment of the time evolution of dynamical systems qua number systems, such as differential (time-continuous) and difference (time-discrete) equations of motion. Even relatively simple nonlinear versions of these systems can give rise to very complex dynamics, including abrupt transitions between different dynamical behaviours when the parameter settings of the equations in question are gradually changed. A key concept in characterizing these dynamical behaviours is that of an attractor: a stable mode of behaviour to which different trajectories in state space (i.e., a representation of the system's behaviour in a coordinate system defined by its state variables) are attracted. Four attractor types are known: (i) the point attractor or equilibrium point, a stable ("steady") single-valued state; (ii) the periodic attractor, a set of state values that are revisited after a fixed time interval, resulting in a stable orbit called a limit cycle; (iii) the quasi-periodic attractor, a set of state values that are not perfectly revisited after a fixed time interval but almost, resulting in a nearly periodic stable orbit, and (iv) the chaotic attractor (or "strange" attractor), a set of state values with no immediately apparent spatiotemporal structure, resulting in an erratic and unpredictable, but stable orbit. Because it is typically impossible to derive analytical solutions of the time evolution of nonlinear systems, the identification of their attractor states and transitions between these attractor states due to parameter variation are crucial steps in the quantitative and qualitative characterization of a particular dynamical system.

In the dynamical systems approach to movement coordination, the concepts and tools of nonlinear dynamics are used to study the stability properties of movement patterns and perception-action patterns. The aim of the approach is to identify principles of coordination that apply generally to pattern formation (i.e., pattern stability and instability) in movement systems with different architectures and structural properties. These principles are identified by mapping observed coordination patterns onto dynamical equations of motion ${ }^{2}$ involving only a limited number of variables that characterize the collective dynamics (i.e., the coordination patterns). The

\footnotetext{
2 The dynamical equation of motion should not be equated with the equations of Newtonian mechanics. The equations of dynamical systems theory refer more generally to any form of time evolution.
} 
resulting dynamical equations of motion of these characteristic variables have the status of "formal analogies" to natural phenomena. The preceding statements reflect four important strategic assumptions of the approach.

(1) Coordination is an a posteriori consequence of pattern formation or physical self-organization (cf. Kelso, 1994a). The notion of self-organization implies that coordinated movements are the orderly products of complex organizations that are composed of a very large number of interacting elements and that may adapt in a flexible manner to changing internal and external conditions by adopting a different coordination pattern without any explicit prescription of this pattern. ${ }^{3}$

(2) General principles of movement coordination exist; that is, there are abstract coordination principles that apply to a variety of movement systems, relatively independent of the structural, material properties of the system's components and of the exact mechanism underlying their interaction. These principles are present at the level of coordinative relations or dynamical actions among things rather than at the level of these things themselves.

(3) Because coordinated movements are the orderly products of physical self-organization, it is both possible and appropriate to describe their intrinsic order in terms of one or a few key variables (cf. Kelso and Schöner, 1988; Schöner and Kelso, 1988c). In synergetics (Haken, 1977; Haken, 1983), as well as other dynamical approaches, these variables are called collective variables or order parameters because they summarize the orderly behaviour that is wrestled out of a large collective of internal degrees of freedom. By definition, these variables are ensemble variables in that they are composed of a number of local variables corresponding to the behaviour of the functional components of the system, i.e., the components over which the order is defined. Closely allied to the synergetic concept of an order parameter is that of the "control parameter": the parameter that affects the number and/or kind of the attractor states of the order parameter without specifying or prescribing those states.

(4) The dynamics of mathematical models of the order parameter(s) formally capture (and correspond to) the behaviour of natural systems,

\footnotetext{
${ }^{3}$ Unfortunately, the notion of self-organization is interpreted by some movement scientists as a kind of mystical ability, according to which movements come out of the blue. This is giving an incorrect ontological twist to the concept.
} 
including qualitative changes therein due to the systematic manipulation of a control parameter. The control parameters have to be identified in conjunction with the collective variables for an adequate account of the observed phenomena. In other words, the aforementioned principles of coordination are considered formalizable in terms of the dynamics of collective variables.

A first and foremost problem in the application of dynamical systems theory to the study a particular phenomenon is to identify one (or a few) appropriate collective variable(s) that capture(s) the order (i.e., the attractor states) of the system. Even though the phenomenon of interest already defines a scale of observation (e.g., neural, electrophysiological, physical, hormonal, behavioural) on which the collective variable(s) is (are) to be sought, there usually still is a wide range of variables that may or may not be essential. Important information about candidate collective variables is provided when the system shifts abruptly from one coordinative mode to another, that is, from one attractor state to another. Such qualitative changes in behaviour, called phase transitions, allow for the identification of collective variables because at these points, where order is lost and regained, it becomes apparent along which dimension the intrinsic order of the system is defined (cf. Kelso, 1994a). Once the collective variable(s) is (are) derived it can be attempted to construct a model in the form of a potential function and/or dynamical equations of motion for the identified collective variable(s). The art of dynamical modelling is to bring out the dynamic richness and complexity of the observed phenomena using a minimal set of formal constructs. Examples of such models will be discussed in the following sections. As we shall see, such models make specific predictions regarding the stable modes of operation as well as the loss of stability near critical points which can be evaluated empirically.

\section{The Haken-Kelso-Bunz-model}

A primary impetus in the development of the dynamical systems approach to movement coordination was provided by the experimental and theoretical work of Kelso and colleagues on the coordination of rhythmic finger (and hand) movements performed at a common frequency of oscillation (Kelso, 1981; Kelso, 1984; Haken et al., 1985). The focus of this work was an abrupt shift in the coordination between two oscillating fingers that can be induced by increasing the frequency of oscillation. When subjects 
are instructed to move their index fingers (or hands) rhythmically in anti-phase (defined as the simultaneous activation of nonhomologous muscle groups) and to gradually increase the cycling frequency, an involuntary abrupt shift to the in-phase pattern (i.e., in which homologous muscle groups are simultaneously active) occurs at a certain critical frequency. Beyond this critical frequency, only the in-phase pattern can be stably performed. Haken et al. (1985) proposed a theoretical model for the abrupt transition in coordinative patterns observed between the hands, using concepts from synergetics and nonlinear oscillator theory. In this section this model (referred to as the HKB [Haken-Kelso-Bunz-model) is discussed.

The first step in modelling this phenomenon involved the detcrmination of the essential variable (or order parameter) and its dynamics. The empirical findings suggested that the relative phase $\phi$ (i.e., the phase difference) between the two oscillating fingers constituted the order parameter, because it characterized the coordinative modes and changed abruptly at the transition. Based on the rather simple dynamics that were observed for $\phi$, its stationary states (i.e., $\phi=0$ and $\phi=\pi$ ) were modelled as fixed point attractors. To account for the observed transition the model had to result in a change from bistability (both $\phi=0$ and $\phi=\pi$ are stable) to monostability (only $\phi=0$ is stable) when the control parameter (movement frequency) reached a critical value. Furthermore, the dynamics had to be $2 \pi$-periodic and, to account for the observed symmetry between the movements of the left and right limb, the model had to be symmetric under the transformation $\phi \rightarrow-\phi$ (so that the description remains the same when the two hands are reversed). According to synergetics, the equations of motion for order parameters are of the form

$$
\dot{\phi}=-\frac{\mathrm{d} V(\phi)}{\mathrm{d} \phi},
$$

where $V(\phi)$ is a potential function that describes the magnitude and the direction of the tendency of $\phi$ to change as a function of its own value and the dot notation represents differentiation with respect to time. If the time-derivative of $\phi$ is zero, the system is in equilibrium. If the equilibrium point is a local minimum of $V(\phi)$, it is stable (i.e., a point attractor), if not, it is unstable (i.e., a repellor). The simplest form of $V(\phi)$ which explains the obtained experimental results proved to be a superposition of two cosine functions:

$$
V(\phi)=-a \cos (\phi)-b \cos (2 \phi) \text {. }
$$



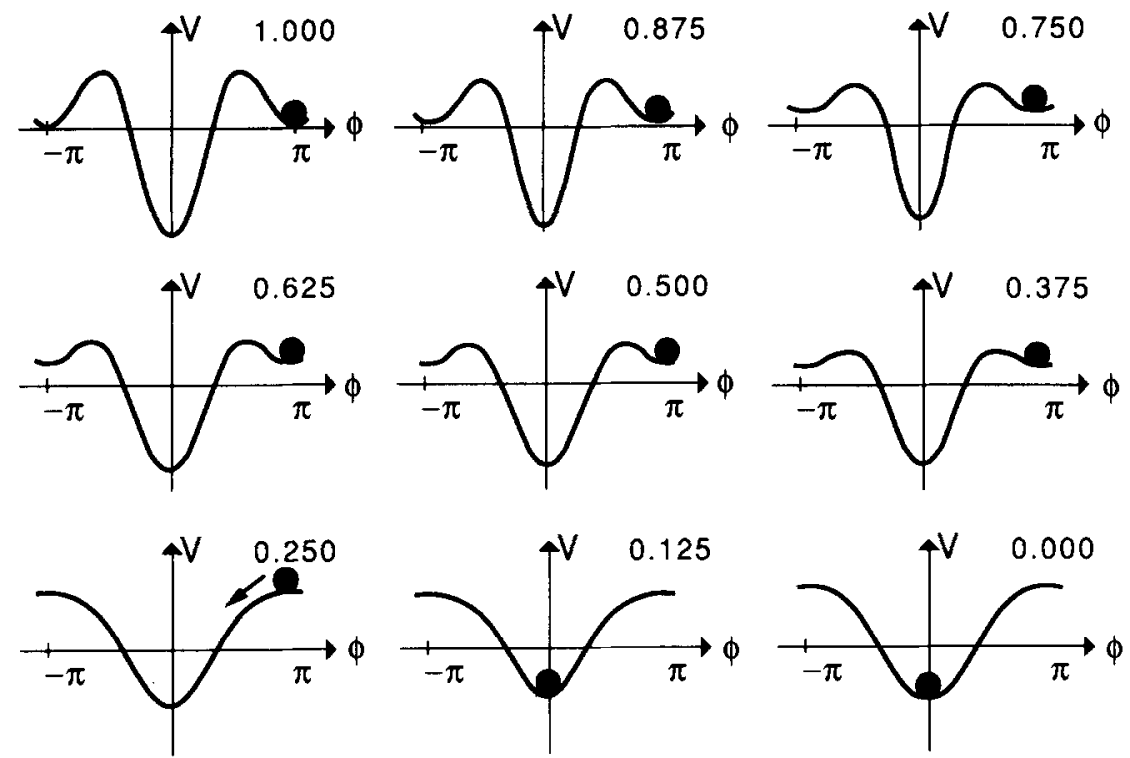

Fig. 1. The shape of the potential $V(\phi)$ for different values of $b / a$ to which the values in the upper right corners of the panels refer (after Haken et al., 1985, adapted with permission. Copyright 1985: Springer-Verlag).

The two cosine functions in Eq. 2 are associated with potential minima at $\phi=0$ and $\phi=\pi$. These minima are the point attractors in the model where the values of the order parameter $\phi$ do not change in time (i.e., $\dot{\phi}=0$ according to Eq. 1). The system is attracted toward one of these values. The stability of the attractors depends on the depth of the minima and the steepness of the slope toward the minimal value, which are both dependent on the ratio between the parameters $b$ and $a$. This is graphically illustrated in Fig. 1 which shows the shape of the potential function for different values of $b / a$. As can be seen, the minimum at $\phi=\pi$ turns in a maximum for $b / a \leq 0.25$. Thus, gradual scaling of the ratio $b / a$ results in loss of stability of the anti-phase coordination followed by a sudden transition to the remaining stable state: in-phase coordination (cf. the black ball in Fig. 1). This implies that the proposed potential function can account for the observed phase transition. In the HKB-model the ratio $b / a$ functions as control parameter: gradual decrease of this ratio induces a sudden transition in the value of the order parameter. Therefore, the increase in movement frequency, which induced the phase transition in the experimental situation, was assumed to result in a decrease of $b / a$. 
The next modelling step involved the derivation of a system of coupled differential equations for the observed movements of the hands of the form

$$
\begin{aligned}
& \ddot{x}_{1}+f_{1}\left(x_{1}, \dot{x}_{1}\right)=I_{12}\left(x_{1}, x_{2}\right), \\
& \ddot{x}_{2}+f_{2}\left(x_{2}, \dot{x}_{2}\right)=I_{21}\left(x_{2}, x_{1}\right) .
\end{aligned}
$$

The left-hand sides of these equations describe the movements of the individual hands in terms of their positions $x_{1}$ and $x_{2}$, respectively, again using the dot notation to indicate time derivatives. The right-hand sides represent the coupling between the hands. The dissipative terms in $f(x, \dot{x})$ are assumed to be weakly nonlinear, involving both positive and negative damping: the component oscillators are self-sustaining and their long-term behaviour is, thus, independent of the initial conditions. When presented in a phase plane (i.e., $\dot{x}$ plotted against $x$ ), the trajectory is attracted to a periodically stable orbit (i.e., a limit cycle). The movements of the individual hands were modelled as a hybrid oscillator, a particular kind of limit cycle oscillator with a nonlinear dissipative structure that accounted for the empirically observed decrease in amplitude and increase in peak velocity with increasing movement frequency. Haken et al. (1985) showed, both analytically and numerically, that this functional form of the individual oscillators in combination with the following coupling function

$$
I_{i j}=\left(\dot{x}_{i}-\dot{x}_{j}\right)\left(\alpha+\beta\left(x_{i}-x_{j}\right)^{2}\right), i=1,2 ; j=2,1
$$

accounts for the observed phase lockings as well as the sudden transition between the coordination modes. In addition, it was demonstrated that the derived system of coupled oscillators was consistent with the derived potential function (Eqs. 1 and 2). ${ }^{4}$ Specifically, the ratio between the parameters in the potential function $(b / a)$ could be related to the parameters $\alpha$ and $\beta$ in combination with the real amplitude of the oscillatory movements, in such a way that a decrease in the amplitude resulted in a decrease in the value of the ratio $b / a$. Given the relation between movement frequency and amplitude in the component oscillators, the role of movement frequency as the control parameter was thus understood.

\footnotetext{
4 The mathematical proof of this step is beyond the scope of this article.
} 


\section{Generalizations of the HKB-model and new directions of research}

Generality of phase transitions. It is important to recognize the intrinsically abstract nature of the HKB-model. Several studies have demonstrated that the model is generalizable to coordinations other than those involving two fingers or hands, such as the coordination between an arm and a leg (Kelso and Jeka, 1992), an arm and a visual stimulus (Byblow et al., 1995; Wimmers et al., 1992) and two legs belonging to different people (Schmidt et al., 1990). These results suggest that the same coordination principles apply to systems with different functional and material components and that the identified coordination dynamics are informational in nature (Kelso, 1994b).

Critical fluctuations and critical slowing down. Immediately following the introduction of the HKB-model the nature of the phase transition was investigated in more detail. Schöner et al. (1986) generalized the model by including stochastic forces that were modelled theoretically as continuous perturbations of the order parameter arising from the microscopic interactions within the system. Because the effects of these stochastic forces are a function of the stability of the attractor state in which the system is prepared, a temporary increase in the resulting deviations away from the attractor state, a phenomenon called critical fluctuations, was predicted to occur when the transition point is approached. These critical fluctuations were indeed observed empirically by Kelso et al. (1986). Also the prediction of a closely related phenomenon, called critical slowing down, was confirmed empirically (Scholz et al., 1987). This is the phenomenon that, prior to a transition, the time it takes the system to return to its stationary state after an external perturbation of the order parameter also increases temporarily due to the loss of stability of the attractor state.

Behavioural information. Another line of investigations on the basis of the HKB-model is concerned with the fact that the coordination dynamics described by the model may change as a function of learning. In an attempt to deal with this phenomenon, Schöner and Kelso (1988a; Schöner and Kelso, 1988b; see also Schöner et al., 1992) distinguished between the already existing pattern dynamics, which they termed "intrinsic dynamics", and the to-be-learned pattern dynamics, which was labelled "extrinsic dynamics". Subsequently, they modelled the learning process as a mapping from the intrinsic dynamics onto the extrinsic dynamics. The to-be-learned dynamical pattern is always specified by behavioural information, i.e., there is always information about the required coordination, be it in the form of 
a perceptual stimulus or in the form of a person's memory or intention. By casting the extrinsic dynamics or behavioural information in the same collective variables that characterize the intrinsic dynamics (e.g., relative phase), it was possible, by combining the intrinsic and extrinsic dynamics, to derive a unified dynamical model for the dynamics of the learning process. Empirical findings with respect to the effects of behavioural information on the pattern dynamics justified this modelling step (Scholz and Kelso, 1990; Zanone and Kelso, 1992).

Oscillators with different eigenfrequencies. The HKB-model was formulated for a symmetrical system, in which the two component oscillators and their eigenfrequencies are identical. However, coordination of different limbs (e.g., the combination of arm and leg movements; see Kelso and Jeka, 1992) involves oscillators with different characteristics such as their eigenfrequencies. The HKB-model was therefore extended to capture the rhythmic coordination of limbs (or, more generally, component oscillators) with different eigenfrequencies (Fuchs et al., 1995; Kelso et al., 1990a; Kelso et al., 1990b). These differences in the eigenfrequencies of the oscillators result in systematic shifts in the location of the attractor states of relative phase in state space (Kelso et al., 1990a,b). The magnitude of these shifts depends on the parametrization of the coupling function, which in turn depends on the movement frequency. The predicted shifts in attractor phase as well as the influence of movement frequency have been empirically demonstrated in anatomically coupled systems (Schmidt et al., 1993; Sternad et al., 1992), as well as in visually and auditorily coupled systems (Kelso et al., 1990a,b; Schmidt and Turvey, 1994). Although inertia and inertial loadings have been the most common way to manipulate the eigenfrequencies of the limbs, it is important to recognize that any influence that causes differences in eigenfrequencies may act as a source of symmetry breaking in the coordination dynamics, including handedness and hemispheric asymmetries (Kelso and Ding, 1993). This shows that although biomechanical properties can shape the coordination dynamics, the principles of coordination dynamics transcend the biomechanical level.

Discrete movements. Although the HKB-model provides a description for the coordination between oscillating limbs, the dynamical analysis of movement coordination did not remain restricted to rhythmic movements. Saltzman and Kelso (1987), for example, showed how discrete movements (such as picking up a cup and bringing it to the mouth) could be modelled as the result of a series of coupled point attractors and limit cycle oscillators corresponding to the joints. In an analogous fashion, Schöner (1990) 
proposed a dynamical model for the coordination of discrete movements in which the starting point and the end point of the movement are modelled as point attractors and the trajectory between them as a part of a limit cycle followed by a transient to the end point. Later, Schöner (1994a; Schöner, 1994b) extended this initial modelling attempt by including the dynamical effect of perceptual information on trajectory formation. These models suggest that discrete movements are amenable to a similar type of dynamical analysis as rhythmic movements (and that there are common principles at work (Schöner, 1994a)) but, to date, they lack the kind of empirical support that is available for dynamical models for rhythmic movement. It remains to be seen whether the difficulties encountered in deriving appropriate dynamical models for discrete movements stem from a principled shortcoming of the approach or not.

\section{Multifrequency tasks}

Another direction in which the dynamical study of movement coordination has been developed is rhythmic multifrequency coordination. We discuss this work in a little more detail because it nicely illustrates the evolution of dynamical models. In a series of recent studies we examined the dynamics of multifrequency coordination in the context of bimanual rhythmic tapping. During multifrequency tapping the two hands tap at different, but fixed, frequencies. The resulting rhythm can be characterized by the ratio between the frequencies at which the hands tap (e.g., 3:2 or 5:3). In general, lower-order ratios (i.e., ratios with smaller numerator and denominator) are performed with less temporal variability than higher-order ratios (e.g., Deutsch, 1983; Summers et al., 1993a; Summers et al., 1993b). In our experimental work we examined this apparent differential stability of rhythmic patterns in relation to the effect of movement frequency. In these experiments skilled male drummers participated, who tapped with their hands on a low resonance tabletop (rotation about the wrist), while their lower arms rested on its surface. One hand had to tap a fixed externally specified frequency, while the other hand had to synchronize to another externally specified frequency that was scaled in a stepwise fashion. Under these experimental conditions, the drummers did not always succeed to perform the externally specified frequency ratio. If the specified ratio could not be performed stably, attraction to a lower-order ratio occurred. These attractions manifested themselves graphically as fre- 
A

Subject C steady period: $600 \mathrm{~ms}$

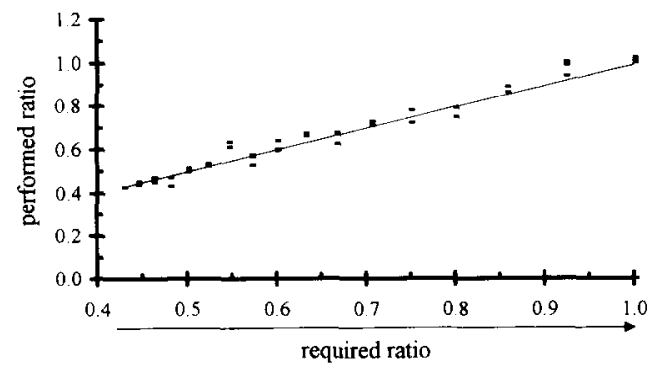

B

Subject C steady period: $\mathbf{4 0 0} \mathrm{ms}$

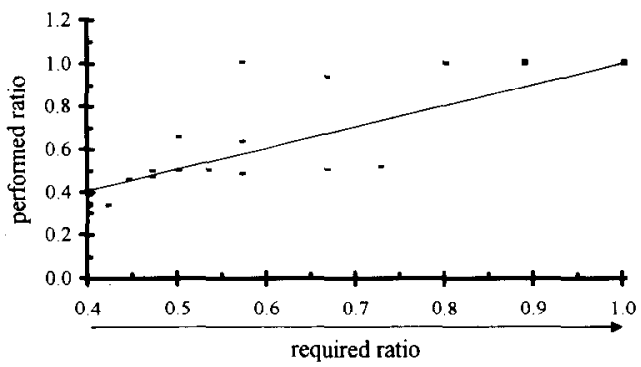

Fig. 2. Stably performed frequency ratios as a function of the externally specified (i.e., required) ratio, as obtained for one of our subjects. In Panel A the ratios were performed slower than in Panel B (indicated by the periods for the "steady" hand). The required frequency of the other hand was increased during the trial. Thus, the required ratio was scaled along the $x$-axis, which is indicated by the arrow. The thin line represents the location of correct responses (i.e., the line $y=x$ ). Comparison of the two panels reveals that the platforms, indicating attraction to lower-order ratios, are larger in the faster condition. (After Peper et al., 1995c, adapted with permission. Copyright 1995: APA.)

quency-ratio platforms in plots of the actually performed frequency ratio against the required frequency ratio (see Fig. 2, Peper et al., 1995c). In general, more attractions to lower-order ratios were observed if the specified frequency ratios had to be performed at higher movement frequencies (compare Fig. 2A versus Fig. 2B).

In another experiment the influence of movement frequency on the performance of multifrequency relations was examined in a more direct manner (Peper et al., 1995c; see also Beek et al., 1992, and Peper et al., 1991). In this experiment the subjects performed either a $8: 3$ or a $8: 5$ frequency ratio, while the movement frequency at which the ratio had to be performed was gradually increased. For all subjects this increase in frequency resulted in a breakdown of performance of the initial ratio, which 


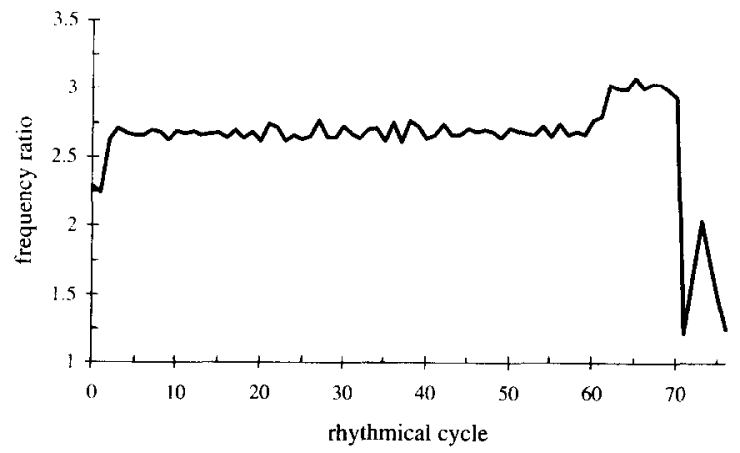

Fig. 3. A typical transition route. The subject performed the frequency ratio $8: 3$. On the $y$-axis the obscrved mean frcquency ratio $\left(f_{\text {right }} / f_{\text {left }}\right)$ per rhythmical cycle (i.c., the period in time in which 8 taps by the right hand and 3 taps by the left hand were to be performed) is presented. Along the $x$-axis the tapping frequency increases in frequency platforms consisting of 3 rhythmical cycles. The figure shows that this increase in movement frequency resulted in a sudden transition from $8: 3$ (i.e., 2.67) to the ratio 3:1 (i.e., 3.0). (After Peper et al., 1995b, adapted with permission. Copyright 1995: Springer-Verlag.)

was, in a majority of trials, followed by a transition to another ratio (see Fig. 3). Analysis of the observed transition routes revealed that almost all transitions were to lower-order ratios. The transitions occurred at movement frequencies that were circa $1 \mathrm{~Hz}$ lower than the maximal tapping frequencies that could be attained unimanually. This implied that the observed transitions resulted from genuine coordination constraints rather than from threshold constraints associated with the separate hands.

The main features of these two experiments, i.e., entrainment (attraction to lower-order ratios) and frequency-induced transitions between coordination patterns, show some clear resemblances to the phase transitions studied by Kelso (1981, Kelso, 1984). Again, a bottleneck appeared in the coordination between the hands, obstructing specific coordination patterns and favouring others. In both experimental tasks, movement frequency acted as a control parameter, in the sense that it guided performance in an unspecific manner from one collective state (i.e., frequency ratio) to another state through loss of stability of the initial state. Some differences with Kelso's work are worth mentioning. First, in our experimental task the observed transition routes were much more diverse than the highly reproducible transition from anti-phase to in-phase observed in Kelso's experiments, revealing that the system operated in a regime in which multiple coordination modes provided stability. Second, multifrequency tasks are hard to perform and, therefore, this behaviour depends to a large degree on practice. Through learning, the subjects acquire a repertoire of coordi- 
nation patterns from which they may intentionally select the to-be-performed ratio. Whereas in the study of 1:1 frequency locking the effects of learning and intention may be addressed as relatively isolated issues (Scholz and Kelso, 1990; Zanone and Kelso, 1992), in the performance of frequency ratios of even relatively low order (e.g., $5: 2$ or $5: 3$ ) the learning history and intentions of the subject play an essential role. In line with the dynamical work on 1:1 frequency coordination, we modelled the tapping hands as a system of coupled oscillators. From this perspective two models proved to be useful for explaining our data. These models differ with respect to the degree of simplification of the rather complex problem of multifrequency coordination. In the next section both models are briefly discussed.

\section{Two dynamical models for multifrequency coordination}

The behaviour of coordinated limb movements which is captured by two coupled differential equations (e.g., the HKB-model), may also be studied by means of a single difference equation in a time-discretized analysis. ${ }^{5}$ In a difference equation the state of the system at time $t+1$ is a function of its state at time $t$. In other words, the state of the system is mapped onto itself or 'iterated'. A general expression of such a difference equation is, thus,

$$
X_{t+1}=F\left(X_{t}\right) .
$$

A system of coupled oscillators, which may be considered a generalized version of the more familiar periodically forced oscillator, can be studied with a special kind of difference equations, called circle maps (cf. Epstein, 1990), which are defined through

$$
\Theta_{n+1}=f_{\Omega}\left(\Theta_{n}\right)=\Theta_{n}+\Omega+g\left(\Theta_{n}\right),
$$

where the variable $\Theta_{n}$ represents the phase of the oscillating system measured stroboscopically (at strobe $n$ ), $\Omega$ is the ratio between the eigenperiods of the forcing and the forced oscillators (i.e., the ratio of the

\footnotetext{
5 In general, difference equations have the advantage that less independent state variables are required to describe a dynamical behaviour of a certain complexity than in differential equations. For example, at least three one-dimensional differential equations are required to create chaos, whereas a single one-dimensional difference equation suffices for generating this type of hehavinur.
} 
periods of the oscillators in the absence of coupling), and $g$ is a periodic function of $\Theta_{n}$ with period 1:

$$
g\left(\Theta_{n}\right)=g\left(\Theta_{n}+1\right)(\bmod 1)
$$

(using the convention of a period of 1 rather than $2 \pi: 0 \leq \Theta<1$ ). As in Eq. 6 the state of the system is mapped onto itself. The phase of the oscillator is influenced periodically ("forced"), which is described mathematically by the forcing function $g\left(\Theta_{n}\right)$ (Eq. 8). The effect of this forcing is a function of the phase of the forced system $\left(\Theta_{n}\right)$. We focus here on the sine circle map, the smoothest of all one-dimensional circle maps, for which the forcing function $g\left(\Theta_{n}\right)$ is defined as $(-)(K / 2 \pi) \sin 2 \pi \Theta_{n}$. During iteration the phase and, hence, the period of the forced oscillator changes due to the coupling effect of the forcing oscillator. The resulting behaviour is characterized by the average ratio between the forcing period and the period of the forced oscillator, the so-called (dressed) winding number, which is defined as $W(K, \Omega)=\left(\Theta_{n}-\Theta_{0}\right) / n$ in the limit as the number of iterations $n$ approaches infinity. Under iteration the variable $\Theta_{n}$ may converge to a series which is either periodic, with $W$ being rational; quasiperiodic, with $W$ being irrational; or chaotic, where the series behaves irregularly. The quantity $W$ is determined by the settings of the parameters of the iteration process: the amplitude (or strength) of the coupling function $K$ and the ratio between the eigenperiods $\Omega$. The system is attracted to a specific periodicity (specified by a rational value of $W$ ) if its parameter values (i.e., $K$ and $\Omega$ ) fall within the boundaries of the associated attraction regime (or "Arnold tongue") as depicted in Fig. 4 (cf. e.g., Hilborn, 1994; Jackson, 1989; Jensen et al., 1984). This implies that the stability of a frequency ratio is associated with the width of the attraction regime. The widths of these regimes vary, implying differential stability of the frequency ratios. In general, lower-order ratios are more stable than higher-order ratios. In addition, Fig. 4 reveals that the widths of the Arnold tongues are a function of the strength of coupling between the oscillators: Stronger coupling is associated with larger stability.

The stability of multifrequency tapping may, thus, be understood in terms of attraction regimes in the sine circle map. The differences in stability observed in the tapping tasks are in general agreement with the identified differential stability of the mode-locked regimes in the sine circle map. To understand the effects of movement frequency, we recall the relation between coupling strength and movement frequency (cf. the HKB-model). Movement frequency has been demonstrated to be inversely 


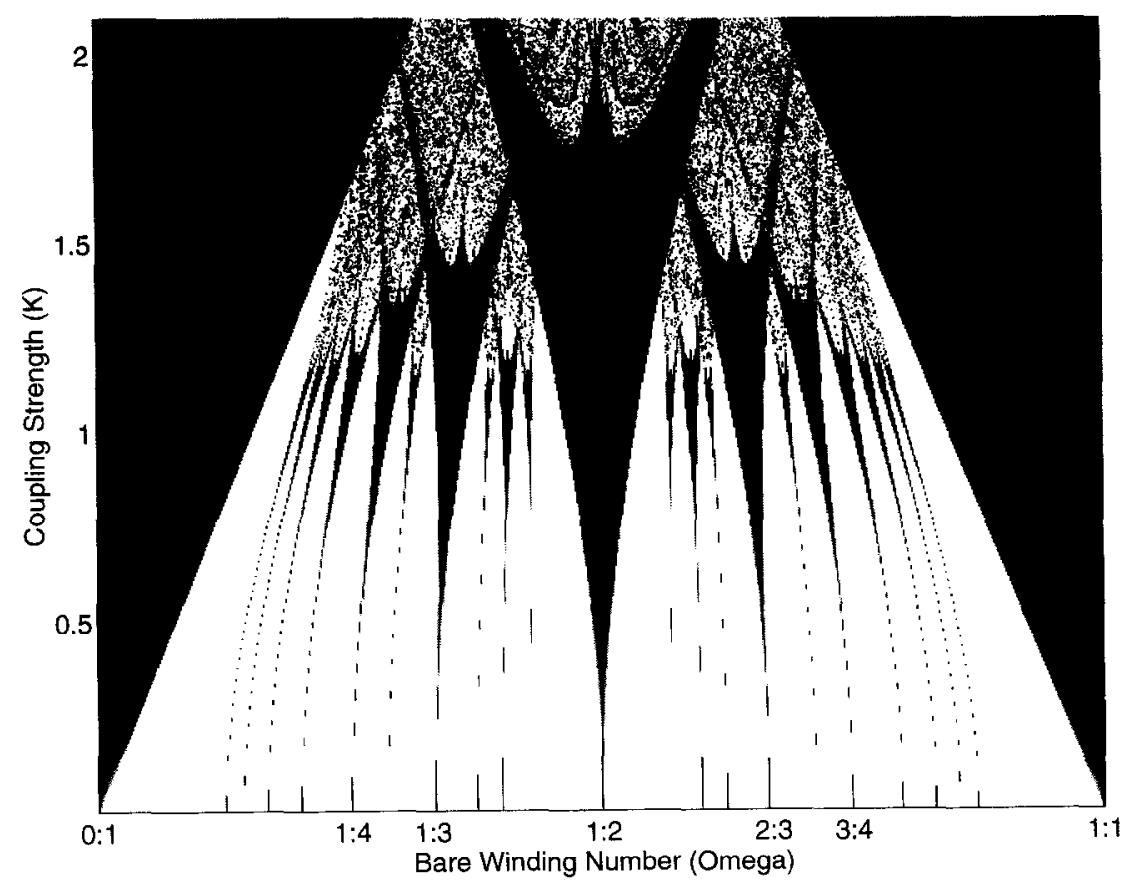

Fig. 4. Regime diagram of the sine circle map which was obtained by performing large numbers of iterations for different combinations of the coupling strength $K$ and $\Omega$, the frequency ratio when the oscillators are uncoupled: the "bare" winding number. The Arnold tongues that demarcate the parameter values of $K$ and $\Omega$ at which the system is attracted to a particular frequency ratio are clearly visible. Darker displays correspond to stronger attraction and the larger a particular range the more stable is the associated mode-locked ratio.

related to the strength of interaction between the oscillating limbs (Peper et al., 1995a; Schmidt et al., 1993; Sternad et al., 1992). This implies that manipulation of movement frequency scales the system along the $y$-axis of the regime diagram depicted in Fig. 4. For higher frequencies (weaker coupling) the attraction regimes are narrower, which is associated with less stable behaviour. Hence, qualitative predictions for the behaviour of coordinated rhythmic movements can be formulated. If movement frequency is increased, stability of the initial coordination pattern may be lost and the system may be attracted to a lower-order ratio that is still sufficiently stable. Because the spatial layout of the attraction regimes is known, the transition routes can also be predicted. In general, our data were in agreement with the predictions based on the sine circle map (Peper et al., 1991; Peper et al., 1995b,c). Data supporting a similar conclusion were reported by Treffner and Turvey (1993). 
The sine circle map provides a rather general account of the behaviour of coupled oscillators. Whereas it has proved an adequate model in understanding the basic phenomena in coordinated multifrequency behaviour (e.g., frequency locking, differential stability of frequency-locked states, and frequency-induced transitions between them), it does not allow for a more detailed examination of the coordination principles. One of the problems in applying this model to our data is that the exact relation between movement frequency and $K$ is unknown. Although converging results indicate that the system is operating in the domain where attraction regimes overlap, resulting in phenomena like multistability and hysteresis (Peper et al., 1995b; Peper et al., 1995c), the precise location of the system in the diagram cannot be determined. $\Lambda$ second problem concerns individual tendencies in the empirically obtained frequency-induced transition routes, which were in some cases even in disagreement with our theoretical predictions (Haken et al., 1995; see also Beek et al., 1992; Peper et al., 1991). The richness observed in multifrequency performance cannot be fully accounted for by this simple map. However, the model may be adapted by modification of the coupling function $g\left(\Theta_{n}\right)$ so that the dynamics of the system change. On the basis of the HKB-model, for example, Kelso et al. (1990a,b; see also deGuzman and Kelso, 1991) formulated a version of the circle map that accounted for the frequently observed tendencies to momentary phase-attraction during multifrequency performance (to the anti-phase or the in-phase mode; e.g., deGuzman and Kelso, 1991; Summers et al., 1993a,b). In order to identify the necessary changes in the coupling function we opted for a similar approach. By constructing a four-dimensional system of differential equations, comparable with the HKB-model, we elaborated on the coupling function in order to gain insight in the influences of learning history and intentions of the individual subject. A number of tools are available to analytically solve nonlinear differential equations (e.g., the rotating wave and the slowly varying amplitude approximations, Haken, 1985). Using this analytically more powerful approach to unravel the mathematical form of the coupling function, we set out to come to terms with the observed individual tendencies in multifrequency performance (Haken et al., 1995).

A new model was developed, based on the same assumptions as the HKB-model. The hand movements were modelled as nonlinear oscillators in a system of coupled differential equations and were identical to those in the HKB-model (the left-hand sides of Eqs. 3). The analysis concentrated on the coupling function through which the equations interact (cf. the 
right-hand sides of Eqs. 3). It was demonstrated theoretically and numerically that specific coupling terms allow for specific frequency locks. Consequently, the observed transitions between frequency ratios imply that the coupling function consists of a large number of coupling terms, each with its own coefficient. These coupling coefficients are associated with the (normalized) amplitudes of oscillation $(r)$ which are raised to a specific power (i.c., associated with $r_{1}^{m} r_{2}^{n}$ ), in combination with a gain parameter $\left(a_{i}\right)^{6}$. Haken et al. (1995) demonstrated that the powers $m$ and $n$ are directly related to the frequency ratio that is supported by the coupling term in question, in such a way that higher powers are present in the coefficients associated with a higher-order ratio. Given the functional form of the component oscillators, increase in movement frequency results in a decrease in the amplitude of the movements. Coupling coefficients which involve higher powers will thus show a stronger decrease than those with lower powers, which is associated with a larger reduction of stability. As a consequence, higher-order ratios loose stability sooner than lower-order ratios, which constitutes the basic mechanism underlying the frequency-induced transitions. In order to stably perform a specific frequency ratio the subject has to (learn to) set the coupling parameter $\left(a_{i}\right)$ associated with the required coupling term. In general, due to the frequency dependence of the amplitudes of the movements, larger values of $a_{i}$ are required for higher-order ratios (involving coefficients with larger powers).

As described above, in the model proposed by Haken et al. (1995) the coupling between the oscillators consists of a large number of coupling terms. The relative weights of these terms are assumed to depend on intrinsic dynamics, learning, and intention. An important feature of this model is that transitions following loss of stability involve detuning of the stiffness parameters. In other words, if stability of the initial pattern is lost, the system "searches" for a new stable solution. In general the detuning will be small, which implies that the newly established ratio will be situated either near the required ratio (on the unit interval) or it may be a ratio with large weights (e.g., a lower-order ratio or an extensively practiced ratio of relatively high order). Although this model was formulated along similar

\footnotetext{
${ }^{6}$ To be specific, the coupling terms were defined as $a_{i} r_{1}^{m} r_{2}^{n} \exp \left[-i\left(m \omega_{1}-n \omega_{2}\right)\right]$ or, alternatively, $a_{i} r_{1}^{m} r_{2}^{n} \sin \left(m \omega_{1}-n \omega_{2}\right)$, where $a_{i}$ is a gain parameter, $r_{1}$ and $r_{2}$ the amplitudes of the slow and the fast hand, respectively, $\omega_{1}$ and $\omega_{2}$ their frequencies, and where the ratio $m: n$ is related to the ratio between these frequencies (e.g., for the coupling from the fast to the slow hand while performing the ratio $p: q$, $m=p \pm 1$ and $n=q$ ).
} 
lines as the HKB-model, some important differences can be singled out: (i) the coupling function consists of a larger number of terms, so that more coordination modes are supported; and (ii) the system is actively involved in the search for stable solutions (i.e., through detuning of the stiffness parameters). Due to the latter two aspects, and in contrast to the task studied by Kelso, marked inter- and intraindividual differences are expected to occur in the transition routes.

The model of differential equations is clearly more complex than the sine circle map. Not only is the dimensionality of the system higher, the coupling function is by far more elaborate. To a large extent the qualitative predictions of the models coincide (e.g., differential stability of frequency ratios, loss of stability when movement frequency is scaled up, and transitions to lower-order ratios). However, certain phenomena can be understood exclusively from the perspective of one of the models. For instance, the circle map readily allows for overlap between frequency locks, resulting in multiple solutions for the same parameter settings (i.e., multistability) a phenomenon that is less easy to study in a nonlinear system of two coupled second-order differential equations. On the other hand, the elaborate coupling function and the possibility for stiffness detuning in the latter model, which provide the starting points for incorporating the influence of learning and intention in the dynamical modelling of multifrequency behaviour, lead to interpretations that are not supported by the sine circle map.

As they stand now, these two models have their own domain of validity and one should avoid making discourse switches within a single analysis. Our purpose of these different lines of modelling, however, was not to arrive at two alternative models, each accounting for a specific subset of the empirically observed phenomena. On the contrary, the goal of these modelling exercises is to develop a unified version. With the help of the analytical tools that are available for systems of differential equations we were able to unpack the coupling function in more detail. The intended future step involves incorporation of this knowledge in a lower-dimensional discrete map, which comprises the explanatory ranges of both models.

\section{Explanatory value of dynamical models}

Now that the reader may have an impression of what the dynamical systems approach to movement coordination is about, and how it has 
matured during the last decade or so into a full-fledged research programme, we are in a better position than before to evaluate the explanatory value of dynamical models for coordination phenomena. We begin our discussion with a quote from Rosenbaum's (1991) book on human motor control that probably summarizes the tacit or explicit appreciation of dynamical systems theory of many movement scientists:

"Synergetics is not a theory of the cause of nonlinearity, however. Nor is it a theory that attempts to say in advance which systems will exhibit which kinds of nonlinearity or what their order parameters or control parameters will be. The theory's aims are purely descriptive, and in that sense modest. On the other hand, synergetics can and does make predictions about individual systems subjected to consistent control-parametcr variations. Phase transitions and critical fluctuations in two-finger oscillations are highly replicable, for example". (Rosenbaum, 1991, p. 372, his italics).

Although this statement is correct in that, as we have seen, dynamical models are mathematical, phenomenological models and not structural models that provide an account of the observed phenomena in terms of their underlying material causes, the statement is theoretically incorrect, or at least biased, in that, as we shall argue shortly, the aims of dynamical systems theory are neither "purely" descriptive in the implied sense that theoretical explanations are absent, nor in any way "modest" (although we must admit that the accusation is an original one). We would like to finesse the assessment of the explanatory value of dynamical systems theory by underscoring the following points.

First, it should be emphasized that dynamical models of the type discussed in this article have to be appreciated in relation to the physical theories of complexity and self-organization in the context of which they are developed. What these theories have in common is the goal to come to terms with the manner in which order arises in complex systems. Because, by definition, these systems have so many elements that it is impossible to describe all the corresponding microscopic degrees of freedom (or microstates), the adopted theoretical strategy has been to describe the patterns of nature at a higher level of abstraction so as to bring out the essential properties of pattern formation. Invariantly, these descriptions are based on specific theoretical ideas about the relationship between the microscopic elements of the system and its macroscopic order. Haken's (1977, 1983) synergetics, for example, postulates a circular causality: the macroscopic order arises out of the nonlinear interactions between the microscopic elements, but governs in turn, once established, the mi- 
crostates (the so-called "slaving principle"). Furthermore, the time scale of events at the macroscale of a complex system (be it a neuron, a brain or a human) is assumed to be different from the time scales of the events at the microscale. When combined, these two ideas not only lead to specific predictions with regard to the transition, such as critical fluctuations and critical slowing down, but also provide a theoretical explanation of these phenomena. Although one can have different opinions as to what extent this explanation is causal, i.e., to what degree it refers to underlying material processes, it certainly adds to the explanatory power of dynamical models such as the HKB-model in that it constitutes a "description of the facts in terms of other facts at another level of experience", the definition provided by Koenderink (1980, p. 390) for "explanation in science". In this sense, Rosenbaum's adjective "purely" in front of "descriptive" is misleading: there are many kinds of explanation besides causal explanations and there are many ways of answering why questions (see Bunge, 1979; Casti, 1991; Van Wieringen, 1988). Also lawful regularities that are not causal may have explanatory value, even, in our opinion, when Koenderink's (1980) somewhat restricted criterion for scientific explanation is not satisfied (cf. Van Wieringen, 1988). Consider for instance the special theory of relativity (cf. Bunge, 1979). The basic axioms of this theory are the invariance of physical laws relative to the choice of inertial systems of reference and the principle of constancy of velocity of light in a vacuum. Neither of these principles is causal in the strict sense of the word, but the special theory of relativity certainly has explanatory power.

In other words, it is difficult to know what exactly Rosenbaum had in mind with his remark that the aim of synergetics qua dynamical systems approach is "purely descriptive", but it seems to us that it is definitely not a pure description of the dynamical enterprise. Clearly, the approach does not simply present the kinematic properties of movement, which would come closest to what we see as pure descriptions, but attempts to account for these properties in terms of theoretical constructs such as potential functions of collective variables.

Another point that has to be emphasized in response to Rosenbaum's statement is that there is no satisfactory theory of the causes of nonlinearity in nature. Even the causal antecedents of seemingly simple transitions from monostable to bistable flow of a gas through a fluid or from laminar flow to turbulence in hydrodynamics are very poorly, if at all, understood. In fact, the limitations inherent to the traditional reductionist programme in explaining natural phenomena have been a strong incentive for abandon- 
ing this programme in favour of a programme aimed at identifying general principles of pattern formation at an abstract level of analysis and to look for communalities in the dynamics of systems with different material constituents. In doing so, theoretical classifications of systems will be developed in the dynamical systems programme of research that might eventually allow one to predict which systems will exhibit which kinds of nonlinearity, which, again, no existing theory is capable of doing.

The previous remarks, of course, do not imply that it is not valid or legitimate to ask why a particular transition occurred once it has been observed. Of course it is. The relevant question however is: what would be a satisfactory answer? This is probably the dimension along which scientists, and especially movement scientists, secm to disagree most. With regard to Kelso's finger experiments, for example, Rosenbaum (1991) proposed the following explanation for the observed transition: Because flexion-extension movements are harder to programme than paired flexion movements and paired extension movements, respectively, it takes longer to initiate the former movements than to the initiate the latter movements. As cycling rate increases, a threshold in the processing of information is reached in that there is not sufficient time any more to initiate flexion-extension movements which causes a transition to a mode of coordination in which the two fingers are flexed and extended simultaneously. That we see something in this explanation is apparent from the fact that it resembles the account we gave for the transition from anti-phase to in-phase coordination while tracking a visual stimulus (cf. Wimmers et al., 1992). Nonetheless, one may ask what exactly is gained by this kind of exercise. A serious problem is that the possible number of information processing models that can be so constructed that they incorporate a phase transition is presumable very large (e.g., the threshold could also be located in the sensory or in the motor channels rather than in the central programming stage, or even in all three of them). Even if we ignore the problem of how to differentiate empirically between these hypothetical models, it will be difficult, in this kind of analysis, to find the most parsimonious information processing model. In the context of the dynamical systems approach, methods are available that can he used (and have been used, for instance, in the derivation of the HKB-model) to find the simplest dynamics that includes all observed intrinsic patterns as well as their instabilities (see, e.g., Guckenheimer and Holmes, 1983). More importantly, however, dynamical models provide a detailed account of all spatiotemporal properties of the observed movement patterns, including the loss of stability and the finger- 
prints of instability near the transition point. While stability and the loss of stability are naturally accounted for by dynamical systems theory, they are not the prime focus of information processing models. At least to us, the lack of theoretical constraints on the construction of motor programmes and the absence of generic concepts to deal with stability-related phenomena renders accounts of phase transitions in these terms not very satisfactory.

In a sense, Van Ingen Schenau et al. (1995) are correct in stating that the discussed phase transitions in interlimb coordination require an internal representation, at least when we assume that they use this term in a broad sense (as we understand they do) to refer to the structural properties of neurons and groups of neurons (or "CPG's") that define the necessary conditions for phase transitions to occur, such as connectivity, synaptic strengths and activity thresholds. This does not imply, however, that the concept of a motor programme is a useful explanatory construct, because this is a very different kind of internal representation, implying a very different approach. Rather than attempting to substitute or complement one phenomenological construct that captures the main features of phase transitions quite well with another construct that captures it less well, it seems a more productive strategy to attempt to uncover the processes that underlie or give rise to specific dynamics. Unlike motor programming approaches, the dynamical systems approach has the advantage that its descriptive apparatus is applicable to phenomena at different scales of analysis, including between-person coordination, perception-action cycles, intra- and interlimb coordination, the brain, subsystems in the brain, small groups of neurons and individual neurons (cf. Beek and Bingham, 1991). With this property the dynamical systems approach is well equipped to deal with the difficult task of linking phenomena at different levels of observation. Examples of how this problem is confronted, and the results it can produce, are provided in the following sections, which discuss the relationships between dynamics and neurophysiology and the relationships between dynamics and biomechanics, respectively.

\section{Neural dynamics}

Neural dynamics have been studied at different levels of organization, such as large collectives of neurons (e.g., the brain), groups of neurons and individual neurons. At the level of large collectives of neurons, the analyti- 
cal tools of dynamics have been instrumental in identifying chaos and other attractor states and changes therein during, e.g., sleep and epileptic seizures (mostly on the basis of the electroencephalogram (EEG); see, e.g., Basar, 1990; Basar and Bullock, 1989; Haken and Koepchen, 1991). Going beyond the mathematical analysis of attractor states (and following Kelso et al., 1991), Fuchs et al. (1992) simultaneously examined pattern formation and switching between self-organized states in sensorimotor coordination and brain activity. They observed the theoretically expected phenomena of critical fluctuations and critical slowing down near the instability point in both the movement kinematics and the magnetically observed brain signals. The brain and behavioural data were subsequently modelled by Jirsa et al. (1994). The derived model, which involved a slightly amended version ${ }^{7}$ of the coupling function of the HKB-model described by Eq. 4, described the main experimental findings very well. According to the authors, the modified HKB-model constitutes a fundamental biophysical coupling for bistable states that applies to all cases in which a switch from one coordinative state to another occurs.

The last-cited study nicely illustrates the advantage of the dynamical systems approach in making connections between the neural level and the behavioural level that was mentioned in the previous section, namely that the mathematical techniques and concepts that are invoked to examine the observed patterns are the same, which is an advantage when integrating results. Order parameters and their dynamics that have been identified and that are empirically established at the behavioural level can be postulated or hypothesized to govern the behaviour of collectives of neurons as well (as has been suggested, e.g., by Kelso et al., 1991). To the extent that this can be demonstrated, it may be possible to derive the collective variables at the behavioural level from the dynamics of the subsystems. Thus, having a good dynamical model of a coordination phenomenon may be helpful in making plausible predictions about the basis of order in the collective states of the brain, and in linking levels of observation.

Also at the level of groups of neurons it is possible in principle to relate the dynamical capabilities of a neur(on)al network (i.e., its nonlinearities and bifurcation conditions) to the architecture of the network (i.e., its connectivity) and the synapse characteristics of the neurons. At least some

\footnotetext{
${ }^{7}$ Specifically, two adjustable constant parameters were added to the coupling described by Eq. 4 to generalize its application to two different kinds of oscillators (i.e., the brain and the stimulus).
} 
modest progress has been made in this regard (see Mpitsos and Soinila, 1993 , p. 272). It is long known that synaptic activation of neurons leads to nonlinear responses due to the firing threshold in the driven neuron. More recently, it is also known how to simulate individual synapses by digital integration, by describing the kinetics mathematically, and by examining the interactions between different kinds of neurons. Much more work needs to be done, however, before the implications of different network architectures and synaptic properties for the collective dynamics of the electrical activity of neurons in these networks will be transparent. Even so, the promise of this work is of great significance: namely achieving a proper theoretical understanding of the pattern dynamics exhibited at the level of groups of neurons in terms of the physiological properties of neurons and their connections. Evidently, the availability of well-established models for the dynamics of neural networks are helpful, if not essential, in understanding the manner in which physiological and architectural properties of neurons and neuronal groups determine these patterns.

An example of the latter statement in the study of movement comes from a series of articles by Collins and Stewart (1992, Collins and Stewart, 1993a,b), who investigated the dynamics of systems of four and six coupled nonlinear oscillators as possible models for locomotor central pattern generators (CPGs) in quadrupeds and hexapods, respectively. They showed some remarkable parallels between the generalities of systems of coupled oscillators and the dynamics of gait patterns, including symmetry properties, symmetry breaking bifurcations (transitions between gaits), and 2:1 frequency-locked oscillations. Natural hierarchies of gaits, ordered by symmetry, and natural sequences of gait bifurcations followed from the mathematical analysis of the model systems. An important implication of this work for the neurophysiological study of CPGs is that the same CPG may control a whole range of apparently very different gaits because small variations in the parameters of the (neural) network are already sufficient to produce different oscillation patterns.

At the level of individual neurons, particularly the study of the dynamics of excitable nerve cell membranes is interesting for the present discussion because it nicely illustrates how dynamical models of a particular behaviour can precede detailed knowledge of microscopic structural elements. This scientific success story dates back to the work of Bernstein (1912), who anticipated, at a time that the instrumentation was lacking to verify so, that neuronal excitation had to depend on changes in membrane permeability. In 1939, Hodgkin and Huxley published the first direct measurements of 
transmembrane potentials in living excitable cells (Hodgkin and Huxley, 1939). These and the rapidly following results triggered an intensive search for a descriptive mechanism behind the phenomenon of propagating action potentials without loss of amplitude, culminating in the nonlinear dynamical Hodgkin and Huxley (1952) model for membrane excitation and propagation. This model, which lent its authors the Nobel prize for medicine in 1963, fitted the experimental data with remarkable accuracy by assuming voltage-dependent membrane permeability mainly for sodium and potassium ions. In the model, the membrane current for sodium depends on the resting voltage for sodium and on the membrane permeability for sodium according to $G_{\mathrm{Na}}=C_{\mathrm{Na}} m^{3} h$, while the membrane permeability for potassium was described by $G_{\mathrm{K}}=C_{\mathrm{K}} n^{4}$ (where $C_{\mathrm{Na}}$ and $C_{\mathrm{K}}$ are constants; Brown, 1984). The parameters $m, h$ and $n$ obey a simple first-order differential equation with voltage-dependent parameters $\alpha_{x}$ and $\beta_{x}$ :

$$
\dot{x}=\alpha_{x}(1-x)-\beta_{x} x,
$$

where $x$ is $m, h$ or $n$ and $\alpha_{x}$ and $\beta_{x}$ arc membrane voltage dependent in an exponential way and differ for $m, h$ and $n$. The kinetics of $m^{3} h$ can be interpreted physically as an ion channel with three identical (fast) and another (slower) gate which must be open simultaneously to let an ion pass. For $n^{4}$ the channel is formed by four identical gates. Hodgkin and Huxley postulated this channel concept without having ever seen one, let alone the specific ion gates. The significance of the Hodgkin-Huxley model for the present context is two-fold. On the one hand, a relatively simple dynamical description was derived for the complex behaviour of a living structure which was later largely confirmed, extended and filled in at a structural molecular level, showing that it is sometimes possible to study the dynamics in connection to the underlying biochemical processes. On the other hand, a way of dealing with membrane dynamics was proposed that later led to the construction of more refined dynamical models (e.g., Chay and Keizer, 1983) and to the experimental identification of empirically relevant dynamical properties, including intermittent beating and chaos (e.g., Chay, 1985; Chay and Rinzel, 1985).

This brief overview of the application of the dynamical systems approach at different neural levels highlights that the more microscopic the level at which a particular behaviour is examined, the better the opportunities are to link the dynamics to structural properties of the system over which these dynamics are defined. This is exemplified by the Hodgkin-Huxley story. In such a case the distinction between phenomenological and structural mod- 
els begins to crumble. Whenever and wherever this can be achieved, an important step is made in science. This, however, is a rare event. Usually, groups of neurons, such as the interneurons in the spinal cord, are already so complex that their collective dynamics cannot be linked to their structural properties. It must be emphasized in this context that the CPGs postulated by Collins and Stewart are a mathematically defined ring of coupled oscillators and not a concrete structure that has been identified as such. In that sense, their model is still fully phenomenological. It needs little further argumentation that at the level of brain activity an understanding of the connection between dynamics and structural properties is remote at best. The strategy from which we expect the most in this regard involves the identification of macroscopic variables and their dynamics at multiple levels of neural organization, and the attempt to link the identified level-specific collective variables across their corresponding levels of observation.

\section{Dynamics and biomechanics}

Unfortunately, the difference between the meaning of the term dynamics in dynamical systems theory and in mechanics has been a persistent source of confusion which has troubled thinking about the relationship between dynamics and biomechanics. In dynamical systems theory, the term dynamics refers to the time evolution of a system at any level of description (e.g., cell, brain or behaviour). In mechanics, in contrast, the term is used as a synonym of kinetics, i.e., the realm of forces and moments, the causes of motion (kinematics) ${ }^{8}$. The difference between the two meanings is exemplified by the observation that it makes sense to talk about the dynamics of brain events but not about the kinetics of brain events. The need to appreciate this difference is amplified when terms like energy, dissipation, stiffness and difference in eigenfrequencies are used in the abstract sense of dynamical systems theory: these terms need not correspond to any observable biomechanical quantities. As has been emphasized by several authors (Kay et al., 1987; Kelso, 1994b; Saltzman and Kelso, 1987), in nonlinear dynamics terms such as stiffness and difference in eigenfrequencies are abstract control parameters that refer to the space-time behaviour

\footnotetext{
${ }^{8}$ In mechanics, the term dynamics is also used as the opposite of statics, which is another distinction than between kinetics and kinematics.
} 
of the system as a whole, whereas in (bio)mechanics they refer to locally identifiable forces and properties of specific structures. To reiterate, an essential assumption of the approach is that, in the study of coordination in complex living systems, appropriate variables at a given level of description exist in the form of collective variables, which are usually not provided by Newtonian mechanics (Kelso, 1994a).

It is probably useful to note in this context that there are some important differences between the dynamical models discussed in the present articles and the equilibrium-point models proposed by Bizzi (1980; Bizzi et al., 1976; Polit and Bizzi, 1979) and Feldman (1966, Feldman, 1986). In the latter kind of models limb movements are understood as the outcome of the qualitative behaviour of a damped mass-spring system with a set of parameters that are adjusted on the basis of control commands from the brain. The goal is to identify the nature of these control commands by analysing the properties of the biomechanical periphery of the motor system to disentangle the aspects of the kinematics that arise from central control and those which arise from the biomechanics and are not controlled directly. To date, dynamical models of movement coordination, in contrast, have typically not been concerned with tying the observed coordination dynamics to the underlying physiological control structures and the biomechanics, at least not in this kind of way. Instead, they were concerned, as we have seen, with the identification of coordination principles that apply quite generally to systems with different neurophysiological and biomechanical properties, which motivated the use of a more abstract language of description.

As we have seen in the example of symmetry-breaking due to differences in eigenfrequencies, biomechanical factors such as moments of inertia, joint compliance, and damping characteristics can shape the coordination dynamics. They do not, however, define the identified principles of coordination; these are defined at a higher level of abstraction and also apply to cases in which variations of the relevant parameters are induced by manipulations and factors other than biomechanical (such as handedness and hemispheric dominance in the case of symmetry-breaking).

In the same way as it was valid for the psychologist to ask why phase transitions occur in bimanual coordination, it is valid for the biomechanicist to ask how biomechanical factors shape the stability properties of movement patterns. In a recent effort to clarify how the macroscopic order of motor patterns is generated from the dynamic interactions between the nervous system, the musculo-skeletal system and the environment, Taga et 
al. (1991) analyzed how the stability properties of walking movements, including transitions between gait patterns, followed from the "global entrainment" between the rhythmic activities of a nervous system composed of coupled neural oscillators and the rhythmic movements of a musculo-skeletal system interacting with the environment. They modelled the neural rhythm generator in terms of a set of differential equations for a network of coupled oscillators and the musculo-skeletal system in terms of a set of Newton-Euler equations and showed how the stable and flexible properties of locomotion followed from the interaction (afferent-efferent coupling) between the two nonlinear dynamical structures. Although the proposed model suffers to a certain degree from the inconsistent use of the term "dynamics" warned against in the preceding, it is highly significant in that it attempts to bridge the gap between the coordination dynamics approach and the more traditional biomechanics-neurophysiology approach to motor control - attempts that have so far been few and far between in the dynamical study of phase transitions and other stability phenomena.

\section{Conclusion}

In the present article we have examined the status of dynamical models of movement coordination. We have seen that the dynamical systems theory offers an approach that can be applied to a variety of disciplines and that generalizes the description of phenomena across a broad range of systems and scales of analysis. The approach allows, in principle, for a rigorous study of complex phenomena such as nonequilibrium phase transitions and multistability. In doing so, it constitutes a generic and empirically progressive research programme. The approach breaks away from the reductionistic tradition in science in postulating order parameters that define a particular level of analysis at which a rigorous, formal characterization of the dynamics is sought. The approach does not provide causal explanations, neither does it provide mere descriptions in the ordinary sense of the word because it is rooted in the mathematical-physical theories of the emergence of order in complex systems.

For movement scientists that are working from other, often more disciplinary, perspectives it may be difficult to appreciate the merits of the dynamical systems approach because its aims, problems, methods and concepts are fundamentally different from those associated with their 


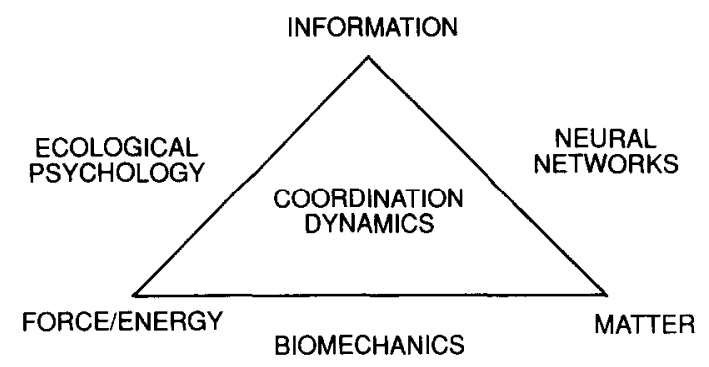

Fig. 5. Tentative scheme for ordering approaches to movement control and coordination.

perspectives. In a final attempt to elucidate these differences we have tentatively constructed the scheme depicted in Fig. 5. The corners of the triangles in this scheme correspond to the primary entities in answering the question how movements come about: information, force/energy and matter. The traditional disciplines of movement science (i.e., (bio)mechanics, psychology, anatomy and physiology) as well as the modern, interdisciplinary approaches in this field of study (i.e., neural networks and dynamics) can be positioned in this scheme. Traditionally, psychology is concerned with information, mechanics with force and energy, and anatomy and physiology with matter (the structural components of the movement system). From each of these corners, attempts have been made to establish meaningful linkages between the three primary concepts. Ecological psychology, for instance, originally an approach predominantly concerned with perceptual information per se (Gibson, 1966, Gibson, 1979), developed into an attempt to link (the pick up of) perceptual information to the control and generation of force and, vice versa, the generation of force to the generation of information (Kugler and Turvey, 1987, see Beek and Van Wieringen, 1994, for an overview). (Bio)mechanics has witnessed a comparable development in that it originally constructed purely mechanical models, such as Hill's muscle model (Hill, 1953), but later attempted to incorporate the structural properties of specific components of the human body, such as striate muscle (cf. Otten, 1991), into these models so as to elucidate the mechanisms for force generation. Conversely, psychologists working in the field of motor control have become increasingly aware of the implications of the biomechanical and physiological properties of the human effector system for the postulation of control structures at a more central level of analysis (e.g., Van Galen and De Jong, 1995). Neural networks, finally, may be viewed as a specific attempt to link information to 
matter, i.e. to understand how information can be represented symbolically in a configuration of neurons (or nodes) and their connection strengths (with all the semantic confusion that one might expect from such an enterprise, cf. Smolensky, 1988). An interesting result of this kind of tentative analysis is that it not only reveals the strong points of these three approaches in movement science but also their limitations. Ecological psychology ignores the problem of how the transactions between information and force in perception-action are supported by neurophysiological processes (matter); biomechanics has a hard time to come to terms with putative control structures (information) that regulate the generation of force by material structures, while connectionism tends to overlook the problem of how the mechanical properties of the musculo-skeletal system (force/energy) are anticipated by the control structures and co-determine the movement outcome.

In Fig. 5, we have positioned dynamics in the middle, not because we believe that it occupies centre stage in the study of movement, but because it transcends the level of description (and abstraction) at which the other approaches operate. Dynamics describes the orderly patterns of behaviour that arise out of the interactions between information, force/energy and matter without, at least at first pass, the aspiration to understand the deterministic relations between these three primary entities and the resulting patterns. Obviously, in movement experiments one can manipulate each of the three identified poles (e.g., by withdrawing or supplying information, by changing the inertial properties of the effector system or the friction between surfaces, and by injecting a chemical substance). Invariantly, these manipulations will affect or shape the resulting pattern dynamics at the coordinative level, but these relations are not straightforward from a modelling point of view. It remains a theoretical challenge to appropriately incorporate the effects of such manipulations in the dynamical models by introducing new terms and/or parameters, and to link the resulting pattern dynamics to dynamical events at more microscopic, neurophysiological levels of observation.

\section{Acknowledgements}

The authors wish to thank Gerard van Galen, Gerrit-Jan van Ingen Schenau, Jeroen Reijnen, Knoek van Soest, Piet van Wieringen, Frank Zaal and an anonymous reviewer for their valuable contributions. Thanks 
are also due to Ed Large for the software to prepare Fig. 4. This research was supported by the Netherlands Organization for Scientific Research (NWO), grant number 575-59-50, and partly supported by NSF Grant SBR 94-22650.

\section{References}

Basar, E. (Ed.), 1990. Chaos in brain function. Berlin: Springer-Verlag.

Basar, E. and T.H. Bullock (Eds.), 1989. Brain dynamics. Berlin: Springer-Verlag.

Beek, P.J. and G.P. Bingham, 1991. Task-specific dynamics and the study of perception and action: A reaction to Von Hofsten (1989). Ecological Psychology 3, 35-54.

Beek, P.J., C.E. Peper and P.C.W. van Wieringen, 1992. 'Frequency locking, frequency modulation and bifurcations in dynamic movement systems'. In: G.E. Stelmach and J. Requin (Eds.), Tutorials in motor behaviour II (pp. 599-622). Amsterdam: North-Holland.

Beek, P.J. and P.C.W. van Wieringen, 1994. Perspectives on the relation between information and dynamics: An epilogue. Human Movement Science 13, 519-533.

Bernstein, J., 1912. Elektrobiologie. Braunschweig: Vieweg und Sohn.

Bizzi, E., 1980. 'Central and peripheral mechanisms in motor control'. In: G.E. Stelmach and I. Requin (Eds.), Tutorials in motor behaviour (pp. 131-143). Amsterdam: North-Holland.

Bizzi, E., A. Polit and P. Morasso, 1976. Mechanisms underlying achievement of final head position. Journal of Neurophysiology 39, 435-444.

Brown, W.F., 1984. The physiological and technical basis of electromyography. Boston: Butterworth Publishers.

Bunge, M., 1979. Causality and modern science. New York: Dover Publications.

Byblow, W.D., R. Chua and G. Goodman, 1995. Asymmetries in coupling dynamics of perception and action. Journal of Motor Behaviour, 27, 123-137.

Casti, J.L., 1991. Searching for certainty. London: Abacus.

Chay, T.R., 1985. Chaos in a three-variable model of an excitable cell. Physica D16, 233-242.

Chay, T.R. and J. Keizer, 1983. Minimal model for membrane oscillations in the pancreatic $\beta$-cell. Biophysical Journal 42, 181-190.

Chay, T.R. and J. Rinzel, 1985. Bursting, beating, and chaos in an excitable membrane model. Biophysical Journal 47, 357-366.

Collins, J.J. and I.N. Stewart, 1992. Symmetry-breaking bifurcation: A possible mechanism for 2:1 frequency-locking in animal locomotion. Journal of Mathematical Biology 30, 827-838.

Collins, J.J. and I.N. Stewart, 1993a. Coupled nonlinear oscillators and the symmetries of animal gaits. Journal of Nonlinear Science 3, 349-392.

Collins, J.J. and I.N. Stewart, 1993b. Hexapodal gaits and coupled nonlinear oscillator models. Biological Cybernetics 68, 287-298.

deGuzman, C.G. and J.A.S. Kelso, 1991. Multifrequency behavioural patterns and the phase attractive circle map. Biological Cybernetics 64, 485-495.

Deutsch, D., 1983. The generation of two isochronous sequences in parallel. Perception and Psychophysics 34, 331-337.

Epstein, I.R., 1990. 'Chemical oscillators and nonlinear chemical dynamics'. In: E. Jen (Ed.), 1989 Lecturcs in complex systems, SFI studies in the sciences of complexity, Lect. Vol. II (pp 213-269). Reading, MA: Addison-Wesley.

Feldman, A.G., 1966. Functional tuning of the nervous system with control of movement or maintenance of a steady posture. II. Controllable parameters of the muscle. Biophysics 11, 565-578. 
Feldman, A.G., 1986. Once more on the equilibrium-point hypothesis ( $\lambda$-model) for motor control. Journal of Motor Behaviour 18, 17-54.

Fuchs, A., V.K. Jirsa, H. Haken and J.A.S. Kelso, 1995. Extending the HKB-model of coordinated movement to oscillators with different frequencies. Manuscript submitted for publication.

Fuchs, A., J.A.S. Kelso and H. Haken, 1992. Phase transitions in the human brain: Spatial mode dynamics. International Journal of Bifurcation and Chaos 2, 917-939.

Gibson, J.J., 1966. The senses considered as perceptual systems. Boston: Houghton Mifflin.

Gibson, J.J., 1979. The ecological approach to visual perception. Boston: Houghton Mifflin.

Guckenheimer, J. and P. Holmes, 1983. Nonlinear oscillations, dynamical systems, and bifurcations of vector fields. New York: Springer-Verlag.

Haken, H., 1977. Synergetics: An introduction. Heidelberg: Springer-Verlag.

Haken, H., 1983. Advanced synergetics. Heidelberg: Springer-Verlag.

Haken, H., 1985. Laser light dynamics. Amsterdam: North-Holland.

Haken, H., J.A.S. Kelso and H. Bunz, 1985. A theoretical model of phase transitions in human hand movements. Biological Cybernetics 51, 347-356.

Haken, H. and H.P. Koepchen (Eds.), 1991. Rhythms in physiological systems. Berlin: Springer-Verlag.

Haken, H., C.E. Peper, P.J. Beek and A. Daffertshofer, 1995. A model for phase transitions in human hand movements during multifrequency tapping. Physica D, in press.

Hilborn, R.C., 1994. Chaos and nonlinear dynamics: An introduction for scientists and engineers. Oxford: University Press.

Hill, A.V., 1953. The mechanics of active muscle. Proceedings of the Royal Society B141, 104-177.

I Iodgkin, A.L. and A.F. I Iuxlcy, 1939. Action potentials recorded from inside a nerve fiber. Nature 144, $710-711$.

Hodgkin, A.L. and A.F. Huxley, 1952. A quantitative description of membrane current and its application to conduction and excitation in nerve. Journal of Physiology 117, 500-544.

Jackson, E.A., 1989. Perspectives of nonlinear dynamics (Vol. 1). Cambridge, UK: Cambridge University Press.

Jensen, M.H., P. Bak and T. Bohr, 1984. Transition to chaos by interaction of resonances in dissipative systems: I. Circle maps. Physical Review A30, 1960-1969.

Jirsa, V.K., R. Friedrich, H. Haken and J.A.S. Kelso, 1994. A theoretical model of phase transitions in the human brain. Biological Cybernetics 71, 27-35.

Kay, B.A., J.A.S. Kelso, E.L. Saltzman and G. Schöner, 1987. Space-time behaviour of single and bimanual movements: Data and model. Journal of Experimental Psychology: Human Perception and Performance 13, 178-192.

Kelso, J.A.S., 1981. On the oscillatory basis of movement. Bulletin of the Psychonomic Society 18, 63.

Kelso, J.A.S., 1984. Phase transitions and critical behaviour in human bimanual coordination. American Journal of Physiology: Regulatory, Integrative and Comparative Physiology 15, R1000-R1004.

Kelso, J.A.S., 1994a. 'Elementary coordination dynamics'. In: S. Swinnen, H. Heuer, J, Massion and P. Casear (Eds.), Interlimb coordination: Neural, dynamical and cognitive constraints (pp. 301-318). New York: Academic Press.

Kelso, J.A.S., 1994b. The informational character of self-organized coordination dynamics. Human Movement Science 13, 415-440.

Kelso, J.A.S., S.L. Bressler, S. Buchanan, G.C. deGuzman, M. Ding, A. Fuchs and T. Holroyd, 1991. 'Cooperative and critical phenomena in the human brain revealed by multiple squids'. In: D. Duke and W. Pritchard (Eds.), Measuring chaos in the human brain. Signapore: World Scientific.

Kelso, J.A.S., G.C. deGuzman and T. Holroyd, 1990a. 'The self-organized phase attractive dynamics of coordination'. In: A. Babloyantz (Ed.), Self-organization, emerging properties and learning (pp. 41-62). New York: Plenun Press.

Kelso, J.A.S., J.D. DelColle and G. Schöner, 1990b. 'Action-perception as a pattern formation process'. In: M. Jeannerod (Ed.), Attention and performance XIII: Motor representation and control (pp. 139-169). Hillsdale NJ: Lawrence Erlbaum Associates. 
Kelso, J.A.S. and M. Ding, 1993. 'Fluctuations, intermittency, and controllable chaos in biological coordination'. In: K.M. Newell and D.M. Corcos (Eds.), Variability in motor control (pp. 291-316). Champaign: Human Kinetics Publishers.

Kelso, J.A.S. and J.J. Jeka, 1992. Symmetry breaking dynamics of human multilimb coordination. Journal of Experimental Psychology: Human Perception and Performance 18, 645-668.

Kelso, J.A.S., J.P. Scholz and G. Schöner, 1986. Nonequilibrium phase transitions in coordinated biological motion: Critical fluctuations. Physics Letters A118, 279-284.

Kelso, J.A.S. and G. Schöner, 1988. Self-organization of coordinative movement patterns. Human Movement Science 7, 2746.

Kugler, P.N. and M.T. Turvey, 1987. Information, natural law, and the self-assembly of rhythmic movement. Hillsdale, NJ: Lawrence Erlbaum.

Koenderink, J. 1980. Why argue about direct perception? Behavioural and Brain Sciences 3, 390-391.

Mpitsos, G.J. and S. Soinila, 1993. 'In search of a unified theory of biological organization: What does the motor system of a sea slug tell us about human motor integration?' In: K.M. Newell and D.N. Corcos (Eds.), Variability and motor control (pp. 225-290). Champaign: Human Kinetics.

Otten, E., 1991. 'Modelling movement control'. In: R. Jacobs and W.E.I. Rikkert (Eds.), Movement control: An interdisciplinary forum (pp. 69-84). Amsterdam: VU University Press.

Peper, C.E., P.J. Beek and P.C.W. van Wieringen, 1991. 'Bifurcations in bimanual tapping: In search of Farey principles'. In: J. Requin \& G.E. Stelmach (Eds.), Tutorials in motor neuroscience (pp. 413-431). Dordrecht: Kluwer.

Peper, C.E., P.J. Beek and P.C.W. van Wieringen, 1995a. Coupling strength in tapping a 2:3 polyrhythm. Human Movement Science 14, 217-245.

Peper, C.E., P.J. Beek and P.C.W. van Wieringen, 1995b. Frequency-induced phase transitions in bimanual tapping. Biological Cybernetics 73, 301-309.

Peper, C.E., P.J. Beek and P.C.W. van Wieringen, 1995c. Multifrequency coordination in bimanual tapping: Asymmetrical coupling and signs of supercritically. Journal of Experimental Psychology: Human Perception and Performance 21, 1117-1138.

Polit, A. and E. Bizzi, 1979. Characteristics of motor programs underlying arm movement in monkeys. Journal of Neurophysiology 42, 183-194.

Rosenbaum, D.A., 1991. Human motor control. New York: Academic Press.

Saltzman, E.L. and J.A.S. Kelso, 1987. Skilled actions: A task dynamic approach. Psychological Review, 94, 84-106.

Schmidt, R.C., C. Carello and M.T. Turvey, 1990. Phase transitions and critical fluctuations in the visual coordination of rhythmic movements between people. Journal of Experimental Psychology: Human Perception and Performance 16, 277-247.

Schmidt, R.C., B.K. Shaw and M.T. Turvey, 1993. Coupling dynamics in interlimb coordination. Journal of Experimental Psychology: Human Perception and Performance 19, 397-415.

Schmidt, R.C. and M.T. Turvey, 1994. Phase-entrainment dynamics of visually coupled rhythmic movements. Biological Cybernetics 70, 369-376.

Scholz, J.P. and J.A.S. Kelso, 1990. Intentional switching between patterns of bimanual coordination depends on the intrinsic dynamics of the patterns. Journal of Motor Behaviour 22, 98-124.

Scholz, J.P., J.A.S. Kelso and G. Schöner, 1987. Nonequilibrium phase transitions in coordinated biological motion: Critical slowing down and switching time. Physics Letters A123, 390-394.

Schöner, G., 1990. A dynamic theory of coordination of discrete movement. Biological Cybernetics 63, $257-270$.

Schöner, G., 1994a. 'From interlimb coordination to trajectory formation: Common dynamical principles'. In: S. Swinnen, H. Heuer, J. Massion and P. Casaer (Eds.), Interlimb coordination: Neural, dynamical, and cognitive constraints (pp. 339-371). New York: Academic Press.

Schöner, G., 1994b. Dynamic theory of action-perception patterns: The time-before-contact paradigm. Human Movement Science 13, 415-440. 
Schöner, G., H. Haken and J.A.S. Kelso, 1986. A stochastic theory of phase transitions in human hand movement. Biological Cybernetics 53, 247-257.

Schöner, G. and J.A.S. Kelso, 1988a. A synergetic theory of environmentally-specitied and learned patterns of movement coordination. I. Relative phase dynamics. Biological Cybernetics 58, 71-80.

Schöner, G. and J.A.S. Kelso, 1988b. A synergetic theory of environmentally-specified and learned patterns of movement coordination. II. Component oscillator dynamics. Biological Cybernetics 58, 81-89.

Schöner, G. and J.A.S. Kelso, 1988c. Dynamic pattern generation in behavioural and neural systems. Science 239, 1512-1520.

Schöner, G., P.G. Zanone and J.A.S. Kelso, 1992. Learning as change of coordination dynamics: Theory and experiment. Journal of Motor Behaviour 24, 29-48.

Smolensky, P., 1988. On the proper treatment of connectionism. Behavioral and Brain Sciences 11, $1-74$.

Sternad, D., M.T. Turvey and R.C. Schmidt, 1992. Average phase difference theory and 1:1 entrainment in interlimb coordination. Biological Cybernetics 67, 223-231.

Summers, J.J., S.K. Ford and J.A. Todd, 1993a. Practice effects on the coordination of the two hands in a bimanual tapping task. Human Movement Science, 12, 111-133.

Summers, J.J., D.A. Rosenbaum, B.D. Burns and S.K. Ford, 1993b. Production of polyrhythms. Journal of Experimental Psychology: Human Perception and Performance, 19, 416-428.

Taga, G., Y. Yamaguchi and H. Shimizu, 1991. Self-organized control of bipedal locomotion by neural oscillators in unpredictable environment. Biological Cybernetics 65, 147-159.

Treffner, P.J. and M.T. Turvey, 1993. Resonance constraints on rhythmic movement. Journal of Experimental Psychology: Human Perception and Performance 19, 1221-1237.

Turvey, M.T., 1990. Coordination. American Psychologist 45, 938-953.

Van Galen, G.P. and W.P. de Jong, 1995. Fitts' law as the outcome of a dynamic noise filtering model of motor control. Human Movement Science 14, 539-571 (this issue).

Van Ingen Schenau, G.J., A.J. van Soest, F.J.M. Gabreëls and M.W.I.M. Horstink, 1995. The control of multi-joint movements relies on detailed internal representations. Human Movement Science 14, $511-538$ (this issue).

Van Wieringen, P.C.W., 1988. 'Kinds and levels of explanation: implications for the motor systems versus action systems controversy'. In: O.G. Meijer and K. Roth (Eds.), Complex movement behaviour: 'The' motor-action controversy (pp. 87-120). Amsterdam: North-Holland.

Wimmers, R.H., P.J. Beek and P.C.W. van Wieringen, 1992. Phase transitions in rhythmic tracking: A case of unilateral coupling. Human Movement Science 11, 217-226.

Zanone, P.G. and J.A.S. Kelso, 1992. The evolution of behavioural attractors with learning: Nonequilibrium phase transitions. Journal of Experimental Psychology: Human Perception and Performance $18,403-421$. 